formalize the plan's lifecycle, identify the decision making team, and determine a deliberative process for decisions during the event. After an initial draft of the plan was prepared, a first stakeholders meeting was held with local law enforcement, the local emergency services agency, and physician and hospital professional organization representatives, who provided valuable feedback. A revised plan is being circulated to county physicians in preparation for the second round of stakeholder review. Following this round, the plan will be finalized and made publicly available.

Results: To date, the plan has been well received; most stakeholders are well aware of the need for such a plan. Recognizing the sensitivity regarding reallocation of limited resources during times of crisis, key stakeholders were involved at multiple levels, which we believe has added to its general acceptance. We are confident the plan will launch successfully after the second round, once clinical decision makers have had an opportunity to provide input. Conclusion: Detailed crisis care planning is feasible at the local jurisdictional level, even with a large population base. Stakeholder involvement and public transparency is essential for the development and implementation of a crisis care plan at the county level.

Prehosp Disaster Med 2017;32(Suppl. 1):s195-s196

doi:10.1017/S1049023X1700512X

\section{Women and Children at Risk for Disasters and ZIKV} Victims in Brazil

Elaine Miranda ${ }^{1}$, Carlos M. Freitas ${ }^{2}$, Vera Pepe $e^{3}$, Dilson

Oliveira ${ }^{1}$, Claudia Osorio-De-Castro ${ }^{4}$

1. Department Of Pharmacy And Pharmacy Administration, Federal Fluminense University, Niterói/Brazil

2. Center For Studies And Research In Disasters And Public Health Emergencies, Fundação Oswaldo Cruz, Rio de Janeiro/Brazil

3. Department Of Administration And Health Planning, Fundação Oswaldo Cruz, Rio de Janeiro/Brazil

4. Department Of Pharmaceutical Policies And Pharmaceutical Services, Fundação Oswaldo Cruz, Rio de Janeiro/Brazil

Study/Objective: To identify health conditions and care for women and children at risk in disasters, and ZIKV victims in selected Brazilian municipalities.

Background: Since the International Health Regulations were re-edited in 2007, four Public Health Emergencies of International Concern (PHEIC) were declared. The most recent was due to the ZIKV outbreak in Brazil. This proposal is based on the assumption that poverty-stricken populations are most vulnerable and especially susceptible to different disasters (epidemics, floods, mud slides etc). Women and children constitute the most vulnerable among the vulnerable, and need close attention and care. PHEICs indicate a need for measures to enhance national preparedness and response capacities, including risk detection and prevention.

Methods: Women in disaster-prone areas or recently affected by disasters, will be identified through their status as beneficiaries of social programs. Each woman will be interviewed as to the nature, possible determinants and consequences of the disaster they experienced, ongoing risks and difficulties faced by them and by children under their care.
Results: We hope to collect relevant information for development and implementation of measures for disaster preparedness and response, regarding these vulnerable groups and especially in relation to PHEIC. This proposal is in line with the perspectives of social inclusion and access to essential rights, thus contributing to reduction of vulnerabilities.

Conclusion: This proposal aims at detecting health care measures for affected and vulnerable groups at-risk for disasters in Brazil, and may establish a benchmark for preparedness and necessary response measures in order to react to present and future PHEIC.

Prehosp Disaster Med 2017;32(Suppl. 1):s196

doi:10.1017/S1049023X17005131

\section{The Effect of Earthquake Hazards Induced by Natural Gas} Mining on Medically Unexplained Physical Symptoms and Psychosocial Problems: A Longitudinal Analysis Michel L.a. Dückers ${ }^{1}$, Joris Yzermans ${ }^{2}$

1. Healthy Communities, NIVEL, Utrecht/Netherlands

2. NIVEL, Utrecht/Netherlands

Study/Objective: To determine whether the chronic threat of exposure to mining-induced earthquakes in the northern part of the Netherlands, is accompanied by a higher prevalence of medically Unexplained Physical Symptoms (MUPS) and psychosocial problems.

Background: The Groningen natural gas field is among the largest in the world and uniquely located in a populated area. Decades of mining activities burdened the region with increased earthquake hazards, several times higher than 3.0 on the Richter scale (one 3.6, in 2012). Inhabitants were confronted with ground shaking, damaged houses, loss of livelihood, social unrest, and public anger towards the mining company and authorities. In 2013 future earthquakes with a magnitude over 5.0 were considered likely, contributing to lack of positive prospects and chronic stress: factors that are not beneficial from a public health perspective.

Methods: Data covering a five-year period (2011-2015), was extracted from electronic patient records of general practitioners. Two dependent variables were constructed: MUPS (physical symptoms clustered per organ system) and psychological and social problems (mood and anxiety disorders, suicide and problems in personal relations or work). Statistical analysis was conducted in steps to test differences in prevalence in health issues in the earthquake region and a comparison group; and to determine the predictive value of gender, age, socioeconomic status, and earthquake magnitude and depth, considering the cross-classified data structure with patients nested in general practices and postal codes. Results: Results: The results indicate whether the prevalence of MUPS and psychosocial problems was significantly higher in the earthquake region regardless of gender and age, after controlling for socio-economic status and earthquake magnitude and depth. Conclusion: The health impact of chronic stress linked to earthquake-threat could be determined apart from other relevant explanatory factors.

Prehosp Disaster Med 2017;32(Suppl. 1):s196

doi:10.1017/S1049023X17005143 